Revista de Derecho

\title{
Delimitación del concepto de riesgo psicosocial en el trabajo
}

\section{Delimitation of the concept psychosocial risk at work}

\section{Elisa Sierra Hernaiz}

Profesora de la Universidad Pública de Navarra

Pamplona, España

elisa@unavarra.es

ORCID: 0000-0002-4320-026X

DOI: https://doi.org/10.32719/26312484.2021.35.1

Fecha de recepción: 30 de junio de 2020

Fecha de aceptación: 22 de septiembre de 2020 


\section{RESUMEN}

En el actual contexto de las relaciones laborales uno de los retos en el ámbito de la prevención de riesgos es el tratamiento de los riesgos psicosociales como riesgos emergentes. Las dificultades que se plantean son muchas, desde la ausencia de un concepto legal de los mismos hasta la incorporación del concepto de salud mental de los trabajadores desde un punto de vista jurídico y no solo psicológico. Sin embargo, ello no puede suponer que estos riesgos queden excluidos de las medidas de prevención de riesgos laborales en la empresa ya que se trata de proteger derechos tan importantes como la integridad física y psicológica o el derecho a no ser discriminado de la persona trabajadora. El tratamiento de estos riesgos en el derecho comunitario europeo y en el derecho español se ha realizado de manera indirecta a través de la normativa preventiva común para todos los riesgos, pero fundamentalmente a través de las Normas Técnicas.

La aprobación del Convenio 190 de la OIT sobre acoso y violencia en el trabajo es un punto de inflexión en esta materia que va a obligar a regular estos riesgos de manera específica, lo que supone un gran paso para su erradicación en el ámbito de las relaciones laborales. Por todo ello, en el presente trabajo se delimita el concepto de riesgo psicosocial como un problema de salud que tiene que tiene que integrarse necesariamente en la política preventiva de la empresa.

Palabras Clave: violencia, acoso, concepto, riesgos psicosociales, trabajo.

\section{ABSTRACT}

Nowadays one of the most important challenges at work is dealing with psychosocial risks at work. The main difficulties are the lack of a legal definition and the incorporation of mental health at work like as a more occupational risk. However, this situation can not mean that these risks are excluded from the policy for risk prevention in the workplace since it is about protecting fundamental rights at work, as the physical and psychological integrity or the right to not be discriminated. Until now the treatment of psychosocial risks at work in European Community law and in Spanish law has been carried out indirectly through the common preventive regulations for all risks, but mainly through the European and Spanish Technical Standards. The adoption of International Labour Organization Convention number 190 recognizes the right of everyone to a world of work free from violence and harassment, including gender-based violence and harassment. This ILO Convention it is a turning point that will force to regulate specifically these risks, which is a great step for its eradication in the field of labor relations. Therefore, in this article the concept of psychosocial risk is delimited as a health problem that must necessarily be integrated into the company's preventive policy

KEYWORDS: violence, harassment, concept, psychosocial, risk, labour. 


\section{INTRODUCCIÓN}

$\mathrm{L}$ os riesgos psicosociales son riesgos emergentes en el ámbito de las relaciones laborales. Se les califica así ya que, a diferencia de los riesgos físicos o biológicos, su inclusión en la normativa preventiva era prácticamente residual hasta tiempos muy recientes, fundamentalmente porque los problemas de salud mental ocasionados como consecuencia de la prestación de servicios por cuenta ajena se consideraba que tenían que ser tratados desde un ámbito médico o por la rama de la psicología laboral. Esta perspectiva cambió hacia los años ochenta del siglo pasado y desde entonces su evolución ha sido hacia su inclusión como un riesgo más dentro de la política preventiva en la empresa. Este cambio es debido a múltiples razones, como, por ejemplo, que el término salud laboral se defina de una manera dinámica; el reconocimiento de que la organización del trabajo y las relaciones personales que se entablan en él pueden producir problemas mentales; un concepto holístico de salud, etc. Por lo tanto, la idea clave es que el desempeño de un trabajo puede afectar negativamente a la salud mental de las personas, desarrollando enfermedades o agravando problemas previos. Si ello es así, ese daño mental también integra la responsabilidad empresarial en materia de prevención de riesgos laborales.

El problema para visibilizar riesgos psicosociales es que no existe una definición del mismo en la normativa de prevención de riesgos laborales, ni en el ordenamiento jurídico comunitario europeo ni español. Ello supone que su delimitación legal se realiza por aproximación a su significado, lo que puede ser un obstáculo para su correcta identificación y erradicación en la empresa. En este contexto han desempeñado un papel fundamental las Normas Técnicas aprobadas por los organismos públicos encargados de velar por la integridad y salud de los trabajadores y, más recientemente, la aprobación por parte de la Organización Internacional del Trabajo (OIT) de su Convenio 190 sobre violencia y acoso, con referencias específicas al entorno laboral por la gravedad de estas conductas en la dignidad de la persona. Pues bien, partiendo de esta realidad en el presente trabajo se delimitará tanto el concepto de riesgo psicosocial como de salud mental desde una perspectiva jurídica para pasar a analizar cuáles son los principales riesgos psicosociales, con especial mención al acoso y violencia en el trabajo.

\section{PUNTO DE PARTIDA: AUSENCIA DE UNA DEFINICIÓN LEGAL DEL CONCEPTO DE RIESGO PSICOSOCIAL}

En la actualidad no existe una definición legal de riesgo psicosocial, lo que, si bien constituye una dificultad para la identificación y erradicación de estas conductas en la empresa, ello no ha obstado para que haya sido incluido dentro de la normativa 
de prevención de riesgos laborales, tanto en el derecho comunitario europeo como español, dado que el concepto de riesgo profesional se configura de una manera muy amplia, al igual que el alcance de la deuda de seguridad del empresario en materia de prevención de riesgos laborales. ${ }^{1}$

Así, por lo que se refiere al derecho comunitario europeo, la norma de referencia es la Directiva marco 89/391/CEE, relativa a la aplicación de medidas para promover la mejora de la seguridad y de la salud de los trabajadores en el trabajo. ${ }^{2}$ Como Directiva Marco su finalidad es fijar unos estándares mínimos comunes para todos los países, comprendiendo dentro de su ámbito todos los riesgos para la salud que tengan su origen en el trabajo sin excepción alguna, fijando las obligaciones de evaluación y adopción de medidas necesarias para evitar o, si no es posible, aminorar los riesgos profesionales. En este sentido, el Tribunal de Justicia de la Unión Europea, en la sentencia de 15 de noviembre de 2001, asunto C-49/00, en sus fundamentos jurídicos números 12 y 13 , recuerda que las obligaciones generales previstas en la normativa, como las de gestionar los riesgos a través de su evaluación y planificación de medidas preventivas, son de directa aplicación a todos los sujetos obligados: ${ }^{3}$

Con carácter preliminar, procede señalar que tanto de la finalidad de la Directiva, que, según su decimoquinto considerando, se aplica a todos los riesgos, como del tenor de su artículo 6, apartado 3, letra a), se desprende que los empresarios están obligados a evaluar el conjunto de los riesgos para la seguridad y la salud de los trabajadores. Es preciso, asimismo, indicar que los riesgos profesionales que han de ser objeto de una evaluación por parte de los empresarios no están determinados definitivamente, sino que evolucionan de forma constante en función, especialmente, del desarrollo progresivo de las condiciones de trabajo y de las investigaciones científicas en materia de riesgos profesionales

En cuanto a la normativa española, en virtud del deber general de protección reconocido en el artículo 4.1.d) del Estatuto de los Trabajadores (ET) y el artículo 14 de la Ley de Prevención de Riesgos Laborales (LPRL), manifestación a su vez del artículo 15 de la Constitución Española (CE), el derecho a la integridad física y psíquica del trabajador ha de ser garantizado en todos los aspectos relacionados con el trabajo, por lo que el empresario está obligado a adoptar cuantas medidas sean necesarias para asegurar su cumplimiento, incluidos los riesgos psicosociales. ${ }^{4}$

1. Véase la Sentencia del Tribunal Supremo Español, de 8 de octubre de 2001, RJ $2002 \backslash 1424$. La jurisprudencia española se puede consultar en la página web del poder judicial. https://bit.ly/2Wo0V1k.

2. DOCE, n. ${ }^{\circ} 183,29$ de junio de 1989.

3. https://bit.ly/3j21Ga9.

4. Elisa Sierra Hernaiz, "Los protocolos de acoso moral y política preventiva de la empresa: puntos críticos y propuestas de mejora", Nueva revista española de derecho del trabajo, n. ${ }^{\circ} 203$ (2017): 95. 
En este sentido, el artículo 4.2 LPRL define el riesgo laboral como: "la posibilidad de que un trabajador sufra un determinado daño derivado del trabajo", cuya gravedad se determinará teniendo en cuenta "la probabilidad de que se produzca el daño y la severidad del mismo". Por lo tanto, la probabilidad y la gravedad de sus consecuencias integran la delimitación legal del riesgo psicosocial, al igual que en el resto de riesgos profesionales. ${ }^{5}$ A su vez, las nociones de daño derivado del trabajo (art. 4.3) y condiciones del trabajo (art. 4.7) de dicha norma hacen posible la inclusión de los riesgos psicosociales.

Pues bien, en una primera aproximación, los riesgos psicosociales son aquellos producidos por la interacción entre los trabajadores y la organización de la empresa y también entre los trabajadores y su entorno social, entendiendo por tal el conjunto de relaciones con los compañeros de trabajo, con los clientes de la empresa o usuarios de los servicios de la misma o cualquier otra relación directa o indirecta que puede surgir durante la prestación de servicios por cuenta ajena. Así, la OIT define los riesgos psicosociales como ${ }^{6}$

las interacciones entre el medioambiente de trabajo, el contenido del trabajo, las condiciones de organización y las capacidades, las necesidades y la cultura del trabajador, las consideraciones personales externas al trabajo que pueden, en función de las percepciones y la experiencia, tener influencia en la salud, el rendimiento en el trabajo y la satisfacción laboral.

En la misma línea, la Agencia Europea de Seguridad y Salud en el Trabajo los delimita como ${ }^{7}$

aquellos aspectos del diseño, organización y dirección del trabajo y de su entorno social que pueden causar daños psíquicos, sociales o físicos en la salud de los trabajadores.

Y, finalmente, la Guía de Actuación Inspectora en factores Psicosociales de la Inspección de Trabajo y de Seguridad Social (ITSS) los configura como ${ }^{8}$

Las condiciones de la relación laboral directamente relacionadas con la organización, el contenido del trabajo, y la realización de la tarea, y por otro las capacidades del trabajador, sus necesidades, su cultura, incluso su situación personal fuera del trabajo, y todo ello en

5. Ibíd., 98 .

6. OIT, Factores psicosociales en el trabajo: Naturaleza, incidencia y prevención (Ginebra, 1986), 12, http:// www.factorespsicosociales.com/wp-content/uploads/2019/02/FPS-OIT-OMS.pdf.

7. https://bit.ly/2WmW40c.

8. ITSS, Guía de actuaciones de la inspección de trabajo y seguridad social sobre riesgos psicosociales (Madrid: Ministerio de Empleo y Seguridad Social, 2012), 13, https://bit.ly/30hui6w. 
cuanto tenga capacidad para afectar tanto al bienestar o a la salud del trabajador como al rendimiento y satisfacción en el trabajo.

De todo lo expuesto se deduce que el concepto de riesgo psicosocial está integrado tanto por elementos objetivos, vinculados a la organización, contenido y realización de la tarea, como por elementos subjetivos, como las capacidades, necesidades, cultura y situación personal del trabajador siempre que afecte a la prestación de servicios. Pues bien, una vez identificado qué son los riegos psicosociales, se hace necesario determinar a qué ámbito de la salud pertenecen para poder evaluados e identificados y establecer las oportunas medidas preventivas para evitarlos o, si no es posible, prevenirlos.

\section{DELIMITACIÓN DEL CONCEPTO DE SALUD EN EL ÁMBITO DE LOS RIESGOS PSICOSOCIALES. LOS FACTORES PSICOSOCIALES Y LOS FACTORES DE RIESGO PSICOSOCIAL}

El término salud y riesgos para la salud de los trabajadores es un concepto amplio y genérico que comprende cualquier riesgo presente o futuro e incluye aspectos físicos, químicos o psicosociales. Así la Organización Mundial de la Salud (OMS) en el preámbulo de su Constitución de 1948 señala: "La salud es un estado de completo bienestar físico, mental y social, y no solamente la ausencia de afecciones o enfermedades". A esta noción también hace referencia el artículo 3.e) del Convenio OIT sobre seguridad y salud de los trabajadores y medio ambiente de trabajo n. ${ }^{\circ} 155$ de 1981: "el término salud, en relación con el trabajo, abarca no solamente la ausencia de afecciones o de enfermedad, sino también los elementos físicos y mentales que afectan a la salud y están directamente relacionados con la seguridad e higiene en el trabajo". De la lectura de ambas normas se deduce que estamos ante una visión integral de la salud en el ámbito laboral, que desde un punto de vista preventivo supone que todos los trabajadores tienen derecho al máximo nivel de protección frente a todos los riesgos laborales, con independencia de su origen, derecho que supone un correlativo deber de seguridad para el empresario que deberá garantizar el máximo nivel de seguridad de sus trabajadores frente a cualquier riesgo laboral, sea cual sea su origen. ${ }^{9}$

9. Sobre la relevancia del concepto de salud en este ámbito concreto véase Sonia Pedrosa Alquezar, "Actualidad de la prevención del acoso moral en el trabajo desde la perspectiva jurídica", en Acoso moral en el trabajo. Concepto, prevención, tutela procesal y reparación de daños, coord. Manuel Correa Carrasco (Pamplona: Aranzadi, 2006), 99 y ss. 
Por lo tanto, si los riesgos psicosociales son riesgos laborales, habrá que fijar a qué ámbito de la salud pertenecen para establecer las medidas preventivas adecuadas. Pues bien, se considera que se integran en el ámbito de la salud mental por los daños psíquicos que pueden producir, aunque también pueden producir problemas graves de salud física, como enfermedades cardiovasculares o problemas músculo esqueléticos. ${ }^{10}$

Una vez concretado en qué ámbito de la salud quedan integrados estos riesgos, el siguiente paso es determinar cuál es su origen. En este contexto, existen dos conceptos de referencia en el ámbito de la prevención de riesgos laborales interrelacionados entres ellos y que son fundamentales en esta materia. El primero es el concepto de factor psicosocial, que hace referencia a las condiciones de trabajo psicosociales y organizativas y se definen como $^{11}$

interacciones entre el trabajo, su medio ambiente, la satisfacción en el trabajo y las condiciones de su organización, por una parte, y por la otra, las capacidades del trabajador/a, sus necesidades, su cultura y su situación personal fuera del trabajo, todo lo cual, a través de percepciones y experiencias, pueden influir en la salud y en el rendimiento y la satisfacción en el trabajo.

Así delimitados, los factores psicosociales tienen su origen, por un lado, en las condiciones organizacionales del trabajo y, por otro, en la percepción y experiencia de los trabajadores de las mismas, esto es, las condiciones sociales en las que se desempeña el trabajo percibidas desde un punto de vista subjetivo. Como tal, estos factores están presente en todas las empresas y no tienen por qué tener siempre connotaciones negativas para la salud de los trabajadores. Así, si la empresa está bien organizada y las condiciones laborales son buenas, pueden producir altos niveles satisfacción en los trabajadores, mientras que si son inadecuados dan lugar a situaciones como, por ejemplo, el estrés. ${ }^{12}$

El segundo concepto de referencia son los factores de riesgo psicosocial, que son aquellos aspectos de la organización del trabajo y de su entorno social que pueden ser la causa de los riesgos psicosociales, puesto que pueden afectar negativamente a la salud y al bienestar de los trabajadores, por lo que actúan siempre como factores desencadenantes de situaciones potenciales de riesgos psicosociales, a diferencia de los

10. Rosa De Luis Aboitiz, "La prevención de los riesgos psicosociales. El procedimiento de gestión del riesgo de violencia en el trabajo" (tesis doctoral, consulta texto original, Universidad Pública de Navarra, 2017), 21 y ss.

11. OIT, Factores psicosociales en el trabajo: Naturaleza, incidencia y prevención, 12.

12. Ibíd., 3. En general sobre esta cuestión véase De Luis Aboitiz, "La prevención de los riesgos psicosociales. El procedimiento de gestión del riesgo de violencia en el trabajo", 18 y ss. 
factores psicosociales. ${ }^{13}$ Se trata, pues, de condiciones de trabajo malas, deficientes o adversas que se convierten en un riesgo laboral. Ejemplo de estos factores son el contenido de trabajo, carga y ritmo de trabajo, tiempo de trabajo, participación y control, desempeño de rol, desarrollo profesional y relaciones interpersonales. ${ }^{14}$

Pues bien, una vez delimitados ambos conceptos existen una serie de situaciones comunes a ambos que se pueden ser fuentes potenciales de riesgos psicosociales y cuya relevancia viene dada por la incidencia que tienen en la empresa, sus efectos en la salud de los trabajadores y las medidas preventivas que se adoptarán. Se pueden clasificar principalmente en tres. ${ }^{15}$

En primer lugar, las relaciones interpersonales en la empresa pueden ser una fuente de conflictos, por lo que necesariamente han de integrar la política preventiva de la empresa, puesto que pueden derivar en situaciones de acoso o violencia en el trabajo.

En segundo lugar, la organización del trabajo puede generar riesgos psicosociales significativos, tanto por el trabajo que se realiza como la forma y condiciones de hacerlo. En este sentido, y a modo de ejemplo, los factores de riesgo psicosocial son el diseño del trabajo por el riesgo que entraña de caer en la monotonía a través de tareas repetitivas o la carga mental de trabajo, por la cantidad y dificultad del mismo, el esfuerzo de atención que requiere o la presión de los tiempos que pueden producir estrés o fatiga mental.

Y, en tercer lugar, el tiempo de trabajo, entendiendo por tal la jornada y el horario de trabajo, que es una de las condiciones laborales con mayor repercusión desde un punto de vista preventivo. Así, como factores de riesgo psicosocial se pueden señalar el trabajo en fin de semana, la falta de descanso semanal, la incompatibilidad de la vida laboral y la vida familiar y personal, la prolongación de la jornada de trabajo, jornadas excesivas de trabajo y especialmente el trabajo nocturno y el trabajo a turnos produciendo también situaciones de estrés o fatiga mental.

\section{ANÁLISIS DE LOS PRINCIPALES RIESGOS PSICOSOCIALES}

Tal y como ha sido señalado en la Introducción, el paso siguiente es el estudio de los principales riesgos psicosociales. De nuevo hay que remitirse a las Notas Técnicas, en el caso del ordenamiento jurídico español, de la Inspección de Trabajo y de la

13. ITSS, Guía de actuaciones de la inspección de trabajo y seguridad social sobre riesgos psicosociales, 14 y ss.

14. Ibíd., 40-1.

15. De Luis Aboitiz, "La prevención de los riesgos psicosociales. El procedimiento de gestión del riesgo de violencia en el trabajo", 18 y ss. 
Seguridad Social y del Instituto Nacional de Seguridad y Salud en el Trabajo (INSST) que identifican como principales riesgos psicosociales el estrés laboral y la violencia en el trabajo. ${ }^{16}$

\section{EL ESTRÉS LABORAL Y SUS MANIFESTACIONES}

En la actualidad, el estrés laboral se considera uno de los riesgos laborales con mayor expansión cuyo origen cabe situarlo tanto en las exigencias del trabajo que no se adaptan a las necesidades, expectativas o capacidades del trabajador como en la falta de compensación adecuada por el trabajo desempeñado. ${ }^{17}$ En cuanto a su alcance, los datos estadísticos revelan que se trata del segundo problema de salud más frecuentemente en el ámbito laboral que se manifiesta principalmente a través del absentismo laboral, tal y como se señala en el Marco estratégico de la UE en materia de salud y seguridad en el trabajo 2014-2020. ${ }^{18}$

Así, el Acuerdo Marco Europeo sobre estrés ligado al trabajo del año 2004 describe el estrés laboral como ${ }^{19}$ "un estado que se acompaña de quejas o disfunciones físicas, psicológicas o sociales y que es resultado de la incapacidad de los individuos de estar a la altura de las exigencias o las expectativas puestas en ellos", indicando que, si bien el estrés no es una enfermedad en sí misma, una exposición prolongada al mismo puede causar problemas de salud por lo que puede terminar convirtiéndose en un riesgo para la salud y seguridad de los trabajadores, generando trastornos de carácter físico, psíquico o conductual cuyo origen puede deberse no solo a factores de riesgo psicosocial sino también a otros agentes como el ruido, vibraciones, elevadas temperaturas, etc. ${ }^{20}$

A su vez, para el INSST el estrés surge

cuando se produce un desajuste entre la persona, el puesto de trabajo y la propia organización, apareciendo cuando el trabajador percibe que no dispone de recursos suficientes para afrontar la problemática laboral. La exposición prolongada a situaciones estresantes en el ámbito laboral y la desadaptación que estas producen en el organismo van a tener como consecuencia más importante la aparición de determinadas enfermedades.

16. ITSS, Guía de actuaciones de la inspección de trabajo y seguridad social sobre riesgos psicosociales, 2.

17. Ibíd., 7.

18. Resolución del Parlamento Europeo, 25 de noviembre de 2015 (2015/2107(INI).

19. https://bit.ly/2B1p725.

20. ITSS, Guía de actuaciones de la inspección de trabajo y seguridad social sobre riesgos psicosociales, 9. 
Respecto a los factores de riesgo psicosocial que generan estrés, y que pueden estar presentes en las condiciones de trabajo, son los estresores del ambiente físico de trabajo; los estresores relativos al contenido de la tarea y, por último, los estresores relativos a la organización. ${ }^{21}$

Dentro del concepto de estrés laboral, y como una manifestación cualificada del mismo, se integra el burnout o desgaste profesional. La importancia de este riesgo queda reconocida desde el mismo momento en que la OMS, en la $72^{\mathrm{a}}$ Asamblea Mundial de la Salud de la OMS, celebrada en Ginebra entre los días 22 y 28 de mayo de 2019, incluye el síndrome de desgaste profesional - expresión que sustituye a la denominación burnt out - como un problema relacionado con el trabajo, siendo una de las novedades más importantes dentro de la undécima revisión de la Clasificación Internacional de Enfermedades (CIE-11). Ello significa que a partir de enero de 2022 el síndrome de desgaste profesional se incluye en el capítulo 24: "Factores que influyen en el estado de salud o el contacto con los servicios de salud" dentro de la subcategoría de "problemas asociados con el empleo y el desempleo" - QD85: Síndrome de desgaste ocupacional—, ${ }^{22}$ lo que contribuirá sin duda alguna a visibilizar los riesgos psicosociales laborales. Así, el síndrome de desgaste ocupacional es definido por el INSST como "una patología derivada de la interacción del individuo con unas determinadas condiciones psicosociales nocivas de trabajo" y es debido a un estrés laboral y organizacional crónico que termina en un estado de agotamiento emocional y de pérdida de motivación laboral para el trabajador debido a las tareas que se ejercen y la falta de condiciones organizacionales que no brindan suficiente protección y apoyo a los trabajadores. ${ }^{23}$

\section{LA VIOLENCIA Y EL ACOSO EN EL TRABAJO}

La violencia se considera el riesgo emergente de carácter psicosocial más importante por todos los derechos y bienes jurídicos afectados con estas conductas, puesto que afecta no solo a la salud, física o psíquica del trabajador sino también a derechos fundamentales, como son la integridad física (art. $15 \mathrm{CE}$ ) o el derecho a no ser discriminado por razón de sexo (art. $14 \mathrm{CE}$ ). En este sentido, los abusos, las agresiones y las amenazas intimidatorias suponen ataques físicos o psicológicos que son susceptibles de causar graves daños y que se manifiestan a través del acoso laboral, el acoso sexual

21. https://bit.ly/3fxMn6S.

22. https://bit.ly/390DMXR.

23. https://bit.ly/3ewIXjo. A su vez, existen dos NTP sobre las consecuencias, evaluación y prevención del burnt out, https://bit.ly/391p7LO y su instrumento de medición https://bit.ly/32jAqh9. 
o la violencia física, ${ }^{24}$ situaciones que son de por sí un riesgo laboral y que, por lo tanto, forman parte de la obligación de seguridad del empresario. ${ }^{25}$

Sus principales manifestaciones son la violencia física y la violencia psicológica, pudiendo ser su origen tanto interno en la empresa - es la que se produce entre compañeros o jefes y subordinados - como externo - derivada tanto de terceros vinculados a la empresa o ajenos a la misma-.${ }^{26}$ En la actualidad se diferencia entre las situaciones de violencia y acoso. Así, el acoso se refiere a una acción continuada de maltrato que sucede de manera reiterada y en varias ocasiones, mientras que la violencia es un acto concreto y puntual de agresión, ya sea física o psicológica, hacia otros compañeros en el trabajo. Esta diferencia también puede ser de intensidad pues mientras en el acoso los actos de amenaza o humillación son leves, pero de manera continuada o repetitiva, en la situación de violencia exige que el acto de agresión tenga una gravedad suficiente para ser considerada como tal. ${ }^{27}$

En la actualidad, la norma de referencia es el Convenio núm. 190 sobre la violencia y el acoso aprobado por la OIT en el año $2019,{ }^{28}$ en cuyo artículo uno define tanto violencia y acoso (a) como violencia y acoso por razón de género (b). Respecto a sus principales novedades en el ámbito laboral cabe destacar dos. En primer lugar, la importancia de este precepto radica en que por primera vez se define un concepto único y mínimo de los conceptos de violencia y acoso, con especial referencia al género, ${ }^{29}$ que deberá ser adaptado a la realidad de cada país que ratifique el Convenio. ${ }^{30}$ De la lectura de ambos apartados cabe destacar que se ha objetivado ambos conceptos, puesto

24. ITSS, Criterio Técnico 69/2009 sobre las actuaciones de la Inspección de Trabajo y Seguridad Social en materia de acoso y violencia en el trabajo (Madrid: Ministerio de Trabajo e Inmigración, 2009), 3.

25. De Luis Aboitiz, "La prevención de los riesgos psicosociales. El procedimiento de gestión del riesgo de violencia en el trabajo", 86.

26. Manuel Velázquez Fernández, "El Convenio 190 de la OIT sobre violencia y acoso en el trabajo: principales novedades y expectativas", Revista de Trabajo y Seguridad Social, CEF, n. ${ }^{\circ} 437-438$ (2019): 127 y ss. así como las Directrices multisectoriales para solucionar la violencia y el acoso de terceros en el trabajo, 2010, https://bit.ly/3iZvIuW.

27. Velázquez Fernández, "El Convenio 190 de la OIT sobre violencia y acoso en el trabajo: principales novedades y expectativas", 126.

28. https://bit.ly/2ZwhwC8.

29. Carmen Grau Pineda, "El reto de la gestión de los riesgos psicosociales y su impacto en el trabajo de las mujeres: la necesaria perspectiva de género", en La gestión del cambio laboral en la empresa, dirigido por Jaime Cabeza Pereiro (Pamplona: Aranzadi, 2017), 100 y ss.

30. Velázquez Fernández, "El Convenio 190 de la OIT sobre violencia y acoso en el trabajo: principales novedades y expectativas", 124. Si bien España aún no ha ratificado dicho Protocolo, la actual ministra de Trabajo anunció en marzo de 2020 su intención de realizarlo con carácter inmediato, lo que aún no ha sido posible debido al actual contexto marcado por el COVID-19 y el estado de alarma que paralizó toda la actividad legislativa. 
que se prescinde del elemento subjetivo de la intencionalidad, y que debe de tratarse siempre de una acción violenta. ${ }^{31}$

a) la expresión "violencia y acoso" en el mundo del trabajo designa un conjunto de comportamientos y prácticas inaceptables, o de amenazas de tales comportamientos y prácticas, ya sea que se manifiesten una sola vez o de manera repetida, que tengan por objeto, que causen o sean susceptibles de causar, un daño físico, psicológico, sexual o económico, e incluye la violencia y el acoso por razón de género, y

b) la expresión "violencia y acoso por razón de género" designa la violencia y el acoso que van dirigidos contra las personas por razón de su sexo o género, o que afectan de manera desproporcionada a personas de un sexo o género determinado, e incluye el acoso sexual.

Y, en segundo lugar, el art. 9 incorpora la prevención de la violencia y el acoso, incluida la violencia y acoso por razón de sexo, como riesgos psicosociales y obliga al empresario a adoptar cuantas medidas sean necesarias para prevenir esas conductas y reparar sus efectos como parte integrante de la política de prevención de riesgos laborales en la empresa. Así:

a) adoptar y aplicar, en consulta con los trabajadores y sus representantes, una política del lugar de trabajo relativa a la violencia y el acoso;

b) tener en cuenta la violencia y el acoso, así como los riesgos psicosociales asociados, en la gestión de la seguridad y salud en el trabajo;

c) identificar los peligros y evaluar los riesgos de violencia y acoso, con participación de los trabajadores y sus representantes, y adoptar medidas para prevenir y controlar dichos peligros y riesgos, $\mathrm{y}$

d) proporcionar a los trabajadores y otras personas concernidas, en forma accesible, según proceda, información y capacitación acerca de los peligros y riesgos de violencia y acoso identificados, y sobre las medidas de prevención y protección correspondientes, inclusive sobre los derechos y responsabilidades de los trabajadores y otras personas concernidas en relación con la aplicación de la política mencionada en el apartado a) del presente artículo.

De todos los riesgos asociados a la violencia y acoso son dos los que presentan mayor relevancia por el impacto que tienen en los derechos fundamentales de los trabajadores, en concreto, a no ser discriminados por razón de sexo y su integridad física, tal y como ya ha sido señalado. Son el acoso moral y el acoso sexual.

31. Ibíd., 125. 


\section{El acoso moral}

El acoso moral es uno de los riesgos emergentes más importantes en la actualidad. ${ }^{32}$ De nuevo, uno de los principales problemas para su identificación y erradicación como riesgo laboral es la ausencia de una noción legal del mismo. ${ }^{33}$ Es cierto que existen definiciones legales en el ámbito de la discapacidad o de la discriminación, pero ello no impide que puedan quedar excluidas situaciones vinculadas a conflictos en el trabajo, que son los que están en el origen de la mayoría de las situaciones de acoso moral. Con todo, se considera que dichas definiciones son de plena aplicación al ámbito de las relaciones laborales en general. ${ }^{34}$

Así, la Ley 51/2003, de 2 de diciembre, sobre igualdad de oportunidades, no discriminación y acceso universal de las personas con discapacidad, Boletín Oficial del Estado (BOE) n. ${ }^{\circ} 289$, define como acoso laboral: "toda conducta relacionada con la discapacidad de una persona, que tenga como objetivo o consecuencia atentar contra su dignidad o crear un entorno intimidatorio, hostil, degradante, humillante u ofensivo" o el concepto del artículo 28 de la Ley 62/2003, de 30 de diciembre, de medidas fiscales, administrativas y del orden social, $\mathrm{BOE} \mathrm{n.}{ }^{\circ} 313$ :

Toda conducta no deseada relacionada con el origen racial o étnico, la religión o convicciones, la discapacidad, la edad o la orientación sexual de una persona, que tenga como objetivo o consecuencia atentar contra su dignidad y crear un entorno intimidatorio, humillante u ofensivo.

Más allá de esta definición, es posible encontrar delimitaciones conceptuales a fenómenos de acoso específico, como el acoso por razón de sexo, definido en el artículo 7 de la Ley Orgánica 3/2007, de 22 de marzo, para la igualdad efectiva de mujeres y hombres (LOI): "constituye acoso por razón de sexo cualquier comportamiento realizado en función del sexo de una persona, con el propósito o el efecto de atentar contra su dignidad y de crear un entorno intimidatorio, degradante u ofensivo". Por lo que respecta al derecho comunitario europeo, el Acuerdo Marco Europeo sobre el acoso y la violencia en el trabajo tampoco proporciona una noción de acoso moral, identificando y describiendo sus formas y manifestaciones, las conductas que integran estos comportamientos o las posibles formas de prevenir, identificar y hacer frente a las situaciones de acoso en la

32. Véase al respecto los datos de las Encuestas de Condiciones de trabajo, tanto europea como española. https://bit.ly/3j4KEbe y https://bit.ly/2WhpBIP.

33. Sobre el enfoque jurídico del concepto de violencia psicológica en el trabajo véase: Manuel Velázquez Fernández, Mobbing. Violencia física y estrés en el trabajo: Aspectos jurídicos de los riesgos psicosociales (Barcelona: Ediciones Gestión, 2004), 39 y ss.

34. María José Romero Rodenas, Protección frente al acoso en el trabajo (Albacete: Bomarzo, 2005$), 10$. 
empresa. ${ }^{35}$ Así, de toda la normativa expuesta se puede extraer un concepto legal básico y general de acoso moral, con independencia de su origen, entendiendo por tal ${ }^{36}$ "toda conducta no deseada... que tenga como objetivo o consecuencia atentar contra la dignidad de una persona y crear un entorno intimidatorio, humillante u ofensivo".

De nuevo son las normas técnicas las que se convierten en referente en esta materia. En el año 2009 el INSST aprobó la Nota Técnica de Prevención (NTP) 854. Acoso Psicológico en el trabajo: definición que define el acoso moral como ${ }^{37}$

La exposición a conductas de violencia psicológica, dirigidas de forma reiterada y prolongada en el tiempo, hacia una o más personas por parte de otra/s que actúan frente aquella/s desde una posición de poder (no necesariamente jerárquica). Dicha exposición se da en el marco de una relación laboral y supone un riesgo importante para la salud.

A su vez, el Criterio Técnico 69/2009, sobre actuaciones de la Inspección de Trabajo y Seguridad Social en materia de acoso y violencia en el trabajo, ${ }^{38}$ sigue la definición de Leymaan contenida en la NTP 476 del INSHT. El hostigamiento psicológico en el trabajo: mobbing según la cual acoso moral $\mathrm{es}^{39}$

una situación en la que una persona o un grupo de personas ejercen una violencia psicológica extrema, de forma sistemática (al menos, una vez por semana), durante un tiempo prolongado (más de 6 meses), sobre otra persona en el lugar de trabajo.

En este contexto de indefinición legal la jurisprudencia de los Tribunales de Justicia ha desempeñado un papel fundamental a la hora de delimitar e identificar estas conductas. Así, el acoso moral consiste en ${ }^{40}$

aquella conducta o pluralidad de comportamientos voluntarios y conscientes de uno o varios sujetos que por su desproporción inciden en otra persona, afectando a su esfera y ámbito personal, suplantando lo que son las relaciones intersubjetivas por afrentas personales o profesionales, puntuales o constantes, que desbordan el ámbito de lo puramente profesional para devaluar al sujeto en su intimidad.

35. Comunicación de la Comisión al Consejo y al Parlamento Europeo por la que se transmite el acuerdo marco europeo sobre el acoso y la violencia en el trabajo, COM/2007/0686 final, https://bit.ly/2B07G1K.

36. Velázquez Fernández, Mobbing, violencia fisica y estrés en el trabajo. Aspectos jurídicos de los riesgos psicosociales, 57.

37. https://bit.ly/30clq21.

38. https://bit.ly/30hcSqR; https://bit.ly/2CFuC6N.

39. https://bit.ly/2Wokpml.

40. Véase, entre otras muchas, la Sentencia del Tribunal Superior de Justicia (STSJ) del País Vasco, de 6 de octubre de 2015 (AS 2016\1065), fundamento jurídico 5.‥ 
De la doctrina jurisprudencial es importante resaltar que el acoso moral exige un plus, la existencia de una situación de hostigamiento del acosador a la víctima, que es precisamente la que hay que objetivar y probar, esto es, demostrar una intención activa por parte del acosador o acosadores de desacreditar profesional al trabajador acosado. $^{41}$

\section{El acoso sexual}

Tanto en el ordenamiento jurídico comunitario europeo como el español, el acoso sexual y el acoso por razón de sexo se definen como comportamientos y conductas discriminatorias mayoritariamente contra las mujeres, aunque puede haber otros colectivos afectados, como los homosexuales o jóvenes. ${ }^{42}$ El punto de partida es la Recomendación de la Comisión Europea de 27 de noviembre relativa a la protección de la dignidad de la mujer y del hombre en el trabajo, considerando al acoso sexual como ${ }^{43}$ "La conducta de naturaleza sexual u otros comportamientos basados en el sexo que afectan a la dignidad de la mujer y el hombre en el trabajo, incluida la conducta de superiores y compañeros". En cuanto a la Directiva 2006/54/CE del Parlamento Europeo y del Consejo de 5 de julio de 2006 relativa a la aplicación del principio de igualdad de oportunidades e igualdad de trato entre hombres y mujeres en asuntos de empleo y ocupación, en su artículo 2.1.c) define el acoso sexual como: "La situación en que se produce cualquier comportamiento verbal, no verbal o físico no deseado de índole sexual con el propósito o el efecto de atentar contra la dignidad de una persona, en particular cuando se crea un entorno intimidatorio, hostil, degradante, humillante u ofensivo". Igualmente, en el ordenamiento jurídico español la LOI en el artículo 7.1 entiende por acoso: "cualquier comportamiento, verbal o físico, de naturaleza sexual que tenga el propósito o produzca el efecto de atentar contra la dignidad de una persona, en particular cuando se crea un entorno intimidatorio, degradante u ofensivo". Por lo que se refiere a las normas técnicas, el Criterio Técnico de la ITSS 69/2000 lo define como ${ }^{44}$

41. Véase al respecto: Manuel Correa Carrasco, "El concepto jurídico de acoso moral en el trabajo", en Acoso moral en el trabajo. Concepto, prevención, tutela procesal y reparación de daños, coord. Manuel Correa Carrasco (Pamplona: Aranzadi, 2006), 67 y ss.

42. Hilda Arbonés Lapena, Violencia en el trabajo: el acoso sexual (Albacete: Bomarzo, 2019), 17 y ss. José Sánchez Pérez, "El acoso sexual y su proyección en las relaciones laborales", Revista de Información Laboral, n. ${ }^{\circ} 8$ (2015): 60 y ss.

43. https://bit.ly/32p67pp.

44. https://bit.ly/2WokAOx. 
todos los comportamientos de naturaleza sexual, tanto aquellos que vayan dirigidos concretamente a una persona concreta (bilateral) ya sea a cambio o no de una determinada exigencia (chantaje) como aquellos de naturaleza sexual que pueden ir dirigidos de forma indeterminada a un colectivo de personas de un determinado sexo a través de escritos, gestos o palabras que pueden considerar ofensivos (acoso ambiental).

Por lo tanto, podría entenderse por acoso sexual en el ámbito laboral como toda conducta de naturaleza sexual, desarrollada en el ámbito de organización y dirección de un empresario o en relación o como consecuencia de una relación de trabajo, cuando la posición ante la misma por parte de la víctima determina una decisión que afecta al empleo o a las condiciones de trabajo de esta o que, en todo caso, tiene como objetivo o como consecuencia, crearle un entorno laboral ofensivo, hostil, intimidatorio o humillante, degradando las condiciones de trabajo de la víctima y poniendo en peligro su salud y su empleo. ${ }^{45}$

Una vez delimitado su concepto, el paso siguiente es concretar los requisitos que se tienen que cumplir para que sea considerado como un riesgo laboral. ${ }^{46}$ En primer lugar, el acoso sexual puede producir daños en la salud de los trabajadores, siendo suficiente que el trabajador quede expuesto a la conducta acosadora sin que sea necesario un daño objetivo e identificado. ${ }^{47}$ En segundo lugar, el acoso sexual se debe ocasionar en el marco de una relación laboral, con una conexión clara con la misma, tal y como entiende el Tribunal Constitucional Español en su sentencia 250/2007, De 17 de noviembre ${ }^{48}$

Al llevarse a cabo, en primer lugar, con ocasión de la prestación del trabajo de la víctima $\mathrm{y}$ del ejercicio por el acosador de las funciones inherentes a su cargo y, en segundo lugar, en el centro de trabajo donde uno y otro coincidían en la realización de sus respectivos cometidos.

En tercer lugar, la conducta ha de tener un carácter indeseado para la persona que lo recibe. ${ }^{49}$ En relación con este requisito, los Tribunales han exigido un rechazo total

45. Teresa Pérez del Río, La violencia de género en el ámbito laboral: el acoso sexual y el acoso sexista. (Albacete: Bomarzo, 2009), 15.

46. De Luis Aboitiz, "La prevención de los riesgos psicosociales. El procedimiento de gestión del riesgo de violencia en el trabajo", 122 y ss.

47. Recomendación de la Comisión Europea, de 27 de noviembre, relativa a la protección de la dignidad de la mujer y del hombre en el trabajo, 4.

48. https://bit.ly/32k8AkY.

49. El INSST, en la NTP 507 Acoso sexual en el trabajo, señala que es importante deslindar las conductas de acoso sexual de las conductas de cortejo, flirteo o comportamiento romántico, https://bit.ly/3h0O90p. 
y absoluto de la víctima respecto de la actitud de sujeto activo. ${ }^{50}$ En cuarto lugar, la conducta puede ser verbal, no verbal o física de naturaleza y connotación sexual, esto es, se trata de conductas de tipo sexual en un sentido amplio. ${ }^{51}$ Se han identificado como conductas verbales las bromas sexuales ofensivas y comentarios sobre la apariencia física o condición sexual del trabajador/a, los comentarios sexuales obscenos, las invitaciones o presiones para concertar citas o encuentros sexuales, etc.; como conductas no verbales, los gestos obscenos, silbidos, gestos o miradas impúdicas, etc., $\mathrm{y}$, finalmente, las conductas de carácter físico, como es pellizcar, tocar, masajes no deseados, etc. ${ }^{52}$ En quinto lugar, el acoso sexual se produce en un entorno de poder en la empresa que conlleva un abuso de autoridad, que supone un chantaje sexual, a través del cual se fuerza a un trabajador/a elegir entre someterse a los requerimientos sexuales o ver perjudicadas sus condiciones de trabajo. En sexto lugar, ha de tratarse de una conducta grave, que atente contra la dignidad de una trabajadora, en particular cuando se crea un entorno intimidatorio, degradante $u$ ofensivo. ${ }^{53}$ Finalmente el acoso sexual no requiere comportamientos reiterados o prolongados en el tiempo, basta una única acción. En cuanto al propósito o intención de provocar daño, la intencionalidad de acosador no es un elemento indispensable. ${ }^{54}$

\section{A MODO DE REFLEXIÓN FINAL}

La aprobación del Convenio OIT 190 refuerza aún más la necesidad de que los ordenamientos jurídicos regulen un marco normativo general para los riesgos psicosociales, al igual que sucede con el resto de riesgos laborales. Aspectos tan importantes como, por ejemplo, delimitar el concepto legal, no solo técnico, de los estresores y sus clases o las distintas formas de acoso laboral, diferenciando entre acoso y hostigamiento dada las distintas implicaciones que tiene cada término es fundamental para asegurar la correcta protección de los trabajadores frente a estos riesgos.

50. Véase la Sentencia del Tribunal Superior de Justicia de Madrid, n. ${ }^{\circ}$ 306/2006, de 27 de marzo.

51. De Luis Aboitiz, "La prevención de los riesgos psicosociales. El procedimiento de gestión del riesgo de violencia en el trabajo", 126.

52. Subdirección General para la Igualdad, Manual de referencia para la elaboración de procedimientos de actuación y prevención del acoso sexual y del acoso por razón de sexo en el trabajo. Aspectos Prácticos (Ministerio de Sanidad, Servicios Sociales e Igualdad, 2015), 11-2, https://bit.ly/2OtDIMg.

53. Véase al respecto la importante Sentencia del TC Español, 224/1999, de 13 de diciembre, https://bit. $1 \mathrm{y} / 2 \mathrm{Zxe} 0 \mathrm{r} 3$.

54. De Luis Aboitiz, "La prevención de los riesgos psicosociales. El procedimiento de gestión del riesgo de violencia en el trabajo", 130. 
A su vez, ese marco normativo tiene que regular con carácter específico todo lo referido a la prevención de riesgos laborales pues se trata de una materia especialmente compleja, ya que cada trabajador puede percibir y, por lo tanto, le pueden afectar estos riesgos de una manera diferente a partir de sus circunstancias personales.

En la actualidad en esta materia deviene fundamental proporcionar respuestas a otros riesgos psicosociales emergentes, como son los producidos por la digitalización, robótica y nanotecnología que dan lugar a lo que se conoce como el tecnoestrés, con factores como la intensificación del trabajo, la prolongación de las jornadas de trabajo, constante disponibilidad, malas posturas, inadecuada iluminación, movimientos repetitivos, posturas forzadas, etc. ${ }^{55} \mathrm{El}$ teletrabajo al que muchos trabajadores se han visto abocados y las condiciones en las que se ha desempeñado como consecuencia del Covid-19 es un ejemplo de ello.

\section{BIBLIOGRAFÍA}

Arbonés Lapena, Hilda. Violencia en el trabajo: el acoso sexual. Albacete: Bomarzo, 2019.

Comisión Europea. Comunicación de la Comisión al Consejo y al Parlamento Europeo por la que se transmite el acuerdo marco europeo sobre el acoso y la violencia en el trabajo, COM/2007/0686 final, https://bit.ly/2B07G1K.

- Comunicación de la Comisión al Consejo y al Parlamento Europeo por la que se transmite el acuerdo marco europeo sobre el acoso y la violencia en el trabajo, COM/2007/0686 final, https://bit.ly/2B07G1K.

—. "Los riesgos psicosociales y el estrés en el trabajo". Agencia Europea para la Seguridad y Salud en el Trabajo. Accedido 30 de junio de 2020. https://bit.ly/2WmW40c.

- Marco estratégico de la UE en materia de salud y seguridad en el trabajo 2014-2020. Resolución del Parlamento Europeo, de 25 de noviembre de 2015 (2015/2107(INI).

- Recomendación de la Comisión, de 27 de noviembre de 1991, relativa a la protección de la dignidad de la mujer y del hombre en el trabajo, DOUE, 24/02/1992. https://bit. ly/32p67pp.

Correa Carrasco, Manuel. "El concepto jurídico de acoso moral en el trabajo". En Acoso moral en el trabajo. Concepto, prevención, tutela procesal y reparación de daños, coordinado por Manuel Correa Carrasco, 41-93. Pamplona: Aranzadi, 2006.

De Luis Aboitiz, Rosa. "La prevención de los riesgos psicosociales. El procedimiento de gestión del riesgo de violencia en el trabajo" (tesis doctoral, consulta texto original, Univer-

55. José Sánchez Pérez, Los riesgos psicosociales en el ámbito laboral: una visión global y práctica (Granada: Comares, 2016), 2 y ss. Véase a modo de ejemplo la guía del Ministerio de Trabajo, Migraciones y Seguridad Social. Robotización y riesgos psicosociales, https://bit.ly/3j6UDNi. 
sidad Pública de Navarra, España, 2017). "Principios ergonómicos relativos a la carga de trabajo mental”, INSST. Accedido 30 de junio de 2020. https://bit.ly/3fxMn6S.

España. "NTP 476: El hostigamiento psicológico en el trabajo: mobbing”. INSST. Accedido 30 de junio de 2020. https://bit.ly/2CFuC6N.

—. "NTP 507: Acoso sexual en el trabajo". INSST. Accedido 30 de junio de 2020. https://bit. ly/2Ww0fa5.

—. 'NTP 704: Síndrome de estar quemado por el trabajo o 'burnout' (I): definición y proceso de generación”. INSST. Accedido 30 de junio de 2020. https://bit.ly/3ewIXjo, 2000.

—. "NTP 705: Síndrome de estar quemado por el trabajo o 'burnout' (II): consecuencias, evaluación y prevención”. INSST. Accedido 30 de junio de 2020. https://bit.ly/391p7LO.

—. 'NTP 732: Síndrome de estar quemado por el trabajo 'Burnout' (III): Instrumento de medición”. INSST. Accedido 30 de junio de 2020. https://bit.ly/32jAqh9.

—. "NPT 854: Acoso psicológico en el trabajo: definición". INSST. Accedido 30 de junio de 2020. https://bit.ly/30clq21.

—. "Riesgos psicosociales: intervención frente al acoso laboral". Ministerio de Empleo y Seguridad Social. INSST. Accedido 30 de junio de 2020. https://bit.ly/3hb3LyM.

—. "Robotización y riesgos psicosociales. Medidas preventivas". Ministerio de Trabajo, Migraciones y Seguridad Social. Accedido 30 de junio de 2020. https://bit.ly/3j6UDN.

-_. "Síndrome del desgaste profesional", INSST. Accedido 30 de junio de 2020. https://bit. ly/390DMXR.

Grau Pineda, Carmen. "El reto de la gestión de los riesgos psicosociales y su impacto en el trabajo de las mujeres: la necesaria perspectiva de género". En La gestión del cambio laboral en la empresa, dirigido por Jaime Cabeza Pereiro, 95-120. Pamplona: Aranzadi, 2017.

ITSS. Criterio Técnico 69/2009 sobre las actuaciones de la Inspección de Trabajo y Seguridad Social en materia de acoso y violencia en el trabajo. Madrid: Ministerio de Trabajo e Inmigración, 2009. https://bit.ly/38XNw56.

- Guía de actuaciones de la Inspección de Trabajo y Seguridad Social sobre riesgos psicosociales. Madrid: Ministerio de Empleo y Seguridad Social, 2012. https://bit.ly/39aiNC9.

OIT. Factores psicosociales en el trabajo: Naturaleza, incidencia y prevención. Ginebra: 1986. https://bit.ly/2WjCFNB.

Pérez del Río, Teresa. La violencia de género en el ámbito laboral: el acoso sexual y el acoso sexista. Albacete: Bomarzo, 2009.

Pedrosa Alquezar, Sonia. “Actualidad de la prevención del acoso moral en el trabajo desde la perspectiva jurídica". En Acoso moral en el trabajo. Concepto, prevención, tutela procesal y reparación de daños, coordinado por Manuel Correa Carrasco, 95-120. Pamplona: Aranzadi, 2006.

Romero Rodenas, María José. Protección frente al acoso en el trabajo. Albacete: Bomarzo, 2005.

Sánchez Pérez, José. "El acoso sexual y su proyección en las relaciones laborales”. Revista de Información Laboral, n. ${ }^{\circ} 8$ (2015): 55-73. 
- Los riesgos psicosociales en el ámbito laboral: una visión global y práctica. Granada: Comares, 2016.

Sierra Hernaiz, Elisa. "Los protocolos de acoso moral y política preventiva de la empresa: puntos críticos y propuestas de mejora". Nueva revista española de derecho del trabajo, n. 203 (2017): 93-123.

Unión Europea. "Directrices multisectoriales para solucionar la violencia y el acoso de terceros en el trabajo". Diálogo Social Europeo. Accedido 30 de junio de 2020. https://bit. ly/3iZviuW.

Velázquez Fernández, Manuel. "El Convenio 190 de la OIT sobre violencia y acoso en el trabajo: principales novedades y expectativas". Revista de Trabajo y Seguridad Social, CEF, n. ${ }^{\circ} 437-438$ (2019): 119-42.

- Mobbing. Violencia física y estrés en el trabajo: Aspectos jurídicos de los riesgos psicosociales. Barcelona: Ediciones Gestión, 2004. 